\title{
CARTOQE : un modèle simplifié pour la gestion de la qualité des eaux des rivières d'un département
}

\author{
J.-P. Merle \\ Ingénieur en chef du G.R.E.F. \\ Service régional d'aménagement des eaux Rhône-Alpes
}

\section{Le problème de l'élaboration des cartes d'objectifs de qualité}

A la suite des résultats peu encourageants de la politique des décrets d'objectifs de qualité de 1971, était engagée par la circulaire du 17 mars 1978 la confection au niveau départemental des cartes d'objectifs de qualité. Ces documents étaient destinés à représenter de façon synthétique les objectifs visés pour la qualité des rivières en fonction des différents usages de l'eau et à servir de cadre à l'action réglementaire des différents services de l'état.

Cette qualité de l'eau était définie par référence à une quarantaine de paramètres physiques, chimiques et bactériologiques, dans les conditions censées être les plus pénalisantes, c'est-à-dire celles de l'étiage.

Une grande latitude était laissée aux différents bassins pour l'application des directives et l'élaboration d'une méthodologie.

Les données sur le milieu dont pouvaient disposer les services étaient extrêmement peu nombreuses, ce qui limita pratiquement les réflexions des services, sauf cas particulier, au traitement de la pollution organique oxydable, les paramètres utilisés pour la confection des cartes de la situation d'origine étant essentiellement la DB05 et accessoirement les teneurs en M.E.S.T., DCO et $\mathrm{NH}_{4}$. Le manque de données sur le milieu pouvait dans une certaine mesure être compensé par l'estimation des rejets. Cette étape était de toute façon indispensable pour définir des objectifs de qualité raisonnables tenant compte des performances des différentes technologies d'épuration.

Très rapidement se posa le problème méthodologique de tenir compte ou non de l'auto-épuration ce qui gêna énormément les services qui souhaitaient avoir du problème une approche quantitative.

C'est dans ce contexte que fut mis au point le logiciel "CARTOQE " qui visait un double but :

1. Proposer aux services départementaux une méthode de travail pour l'élaboration de leur carte en les soulageant de calculs fastidieux.

2. Leur donner la possibilité de tenir compte de l'autoépuration en effectuant des calculs impensables avec les calculettes de bureau.

\section{Le logiciel CARTOQE}

L'objectif du logiciel était à partir de données caractéristiques des rejets d'en déduire la qualité des cours d'eau. Pour ce faire, il utilisait un certain nombre de données dont la plupart était censée être connue des services :

- les paramètres caractéristiques des rejets et de leur évolution dans le temps. Le logiciel a la capacité de gérer une dizaine de paramètres, mais dans la pratique, seuls 2 ou 3 étaient utilisés.

CARTOQE : a simplified model to manage the quality of water in the rivers of a Department

The software CARTOQE has been designed to help with the making of quality objective maps for the Departments in the Region. Five Departments have used it to date. It works like a simplified model likely to represent trends in the quality of the water in reference periods of water flow rates in a river, and takes into account : pollutated water waste, transmission coefficients for the pollution; (representative of autopurification) for the various parameters characteristic of the quality of the water; the "background noise", that is the natural contents of water for the various parameters. 
Pour chacun de ces paramètres il était possible de définir plusieurs états dépendants de différentes hypothèses concernant les niveaux de traitement. Le plus souvent, 3 hypothèses étaient retenues: celle correspondant à la situation d'origine, permettant de restituer en principe le constat de la situation de départ tel qu'il avait été fait par le groupe de travail ad hoc, et 2 situations correspondant à des niveaux de traitement envisageables pour la situation objectif ;

- les paramètres caractéristiques du cours d'eau définis par tronçons homogènes :

- le débit de référence : était le plus souvent choisi le débit mensuel d'étiage quinquennal,

- le bruit de fond par paramètre : c'est-à-dire la concentration des différents parmètres que l'on pourrait qualifier de «naturelle » et que l'on pourrait mesurer en l'absence de pollution,

- le coefficient de transmission de la pollution : ce coefficient permet de tenir compte de l'auto-épuration et peut être fixé par paramètre. Bien entendu, sa signification n'est entière que pour les paramètres caractéristiques de la pollution oxydable. Cependant, pour d'autres paramètres, on constate également une décroissance des teneurs à l'aval des points d'émission que l'on peut en quelque sorte assimiler, avec prudence naturellement, à une auto-épuration. C'est le cas en particulier du paramètre ammonium dont l'évolution est également commandée par des processus biologiques.

\section{La mise en auvre de CARTOQE}

Le logiciel utilise deux fichiers; le fichier des rejets dont la position sur le cours d'eau est définie par leur $p K$ et le fichier des sections de cours d'eau, dont la numérotation permet de rendre compte de l'arborescence des cours d'eau.

Les calculs de transmission de la pollution entre deux sections sont faits à l'aide de l'expression :

$$
F n=F n-1 \cdot K^{\Delta \mathrm{pK}}
$$

dans laquelle :

- Fn et $F n-1$ sont les flux polluants au niveau de 2 sections de cours d'eau. La section n étant à l'aval de la section $n-1$ et les calculs se faisant de l'amont vers l'aval ;

- $K$ le coefficient de transmission de la pollution sur la section de cours d'eau considérée. Ce coefficient est inférieur à 1 . Pris égal à 1 , il exprime la transmission intégrale des flux de pollution;

- $\Delta \mathrm{pK}$ est la distance qui sépare les sections $N$ et $N-1$.

Si des rejets existent entre les deux sections, l'impact de leur pollution au point $N$ est calculé de manière analogue.

En rapportant les flux obtenus aux débits de référence, en ajoutant le bruit de fond, on obtient les concentrations permettant pour chaque paramètre de déterminer la classe de qualité.

Le problème est donc de déterminer le coefficient $K$. Pour ce faire, 2 possibilités existent: le mesurer sur le terrain ou l'utiliser comme coefficient de calage du modèle.
La mesure du coefficient de transmission nécessite sur le terrain des manipulations relativement lourdes : il convient de disposer de tronçons relativement longs, de faciès homogène, indemnes de rejets si possible et d'opérer dans des conditions les plus proches possibles du débit de référence. De telles conditions ont pu être obtenues dans quelques études (cf. Etude du Contrat de rivière Ardèche Claire). Mais, il n'était bien entendu pas question, dans le cadre de l'élaboration des cartes d'objectifs de qualité, de mettre en œuvre systématiquement de tels dispositifs. Aussi, a-t-on le plus souvent déterminé $K$ par tatonnements, l'opérateur étant guidé par le faciès de la rivière : - plus la rivière est lente, plus le coefficient est petit (pouvoir auto-épurateur élevé à condition que la capacité d'oxygénation ne soit pas le frein);

- les rivières ensoleillées ont une capacité auto-épuratrice plus importante que les rivières ombragées ;

- les rivières à étiage hivernal ont une capacité autoépuratrice faible en raison de la température qui ralentit les processus biologiques.

- etc...

Après un essai de quelques jeux de coefficient par bassin versant, l'opérateur parvient en général à reconstituer l'image de la situation d'origine ou à retrouver les flux des différents paramètres polluants qui auraient pu faire l'objet de mesures. Ayant ainsi déterminé ces coefficients de calage, l'opérateur est prêt à simuler l'impact des différents scénario qu'il a bâti pour les différents rejets.

\section{Les résultats}

La méthode CARTOQE a en région Rhône-Alpes été utilisée par les départements qui en 1982 n'avaient pas encore mis au point leurs objectifs de qualité : l'Ardèche, l'Isère, le Rhône, la Savoie et la Haute-Savoie.

Elle exige une bonne connaissance des rejets et de ce fait a permis dans certains cas, de réviser quelques conclusions du constat initial de la qualité, qui dans beaucoup de départements avait été élaboré quelques années auparavant.

A titre d'exemple, le tableau ci-contre résume le travail fait en Haute-Savoie pour la mise au point des objectifs de qualité du Fier sur le paramètre DB05.

La première colonne cumule simplement les flux des rejets polluants et apporte la démonstration que l'hypothèse négligeant l'auto-épuration, ne permet pas de rendre compte de la réalité exprimée dans la colonne 3 , où sont consignés les résultats synthétiques d'une campagne de mesures.

La deuxième colonne donne les résultats des calculs effectués avec les coefficients choisis par l'opérateur de 0,9 pour les affluents du Fier et 0,95 pour le Fier lui-même. Bien entendu, les écarts exprimés en flux avec les mesures sont encore importants mais cependant admissibles, compte tenu de l'imprécision de la mesure du paramètre considéré pour lequel, dans la gamme des concentrations obtenues, 0,2 à $0,4 \mathrm{mg} / \mathrm{l}$ est une bonne fourchette d'incer- 
titude. Il aurait sans doute été possible d'améliorer la coïncidence en affinant la détermination de $K$. Dans la pratique, les coefficients de transmission de la pollution choisis varient de 0,75 (soit un abattement de $25 \%$ de la pollution sur $1 \mathrm{~km}$ ) à 0,95 ou même 1 .

\section{Les limites de la méthode CARTOQE}

L'outil CARTOQE a été confectionné pour mettre au point les cartes d'objectifs de qualité dans un cadre cohérent. Son utilisation dans des conditions fortement différentes de celles choisies risque par conséquent de générer des déboires d'autant plus pernicieux qu'un programme de calcul donne toujours des résultats. En particulier, il convient de se garder de l'utiliser dans des gammes de débit s'écartant fortement du débit de référence, ou pour des paramètres pour lesquels l'auto-épuration n'aurait pas de signification.

\section{Utilisation des débits différents du débit de référence}

L'expression utilisée pour le calcul est dérivée de celle du modèle de Streeter et Phelps qui peut s'écrire :

$$
F=F \mathrm{o} .10^{-\mathrm{K} 1 \times \cdot \mathrm{t}}
$$

dans laquelle : $K I$ est le coefficient de biodégradation, et $\mathrm{t}$ le temps.
Les temps de transfert n'étant pas connus, pour des raisons de facilité, on a remplacé cette variable par $\Delta p K$, une distance, facilement mesurable sur une carte. Le coefficient $K$ correspondant aux conditions de l'étiage n'a donc aucune raison d'être le même que le coefficient correspondant à d'autres conditions de débit, il a même toutes les raisons d'être différent et plus élevé (moins d'autoépuration entre 2 points) si le débit augmente compte tenu du raccourcissement du temps de transfert.

\section{L'extension à d'autres paramètres que la DBO5}

Ayant constaté que de nombreux paramètres caractéristiques de la pollution décroissaient à l'aval des rejets, soit pour cause de transformation biologique, soit pour des raisons tenant à la sédimentation active dans les conditions choisies pour la détermination des objectifs de qualité, la tentation est grande d'appliquer à ces paramètres un traitement analogue à celui de la DBO5.

Une telle extension ne peut se faire qu'avec beaucoup de précautions et en conservant en permanence à l'esprit les limites de la méthode. Elle paraît cependant à peu prêt licite pour des paramètres comme l'ammonium, très souvent déclassant pour les rivières recevant une forte charge organique. Cependant, s'il a été possible de vérifier le bien-fondé d'une telle entreprise dans des cas de pollution modérée, il convient d'être beaucoup plus circonspect si la charge organique augmente.

\begin{tabular}{|c|c|c|c|c|c|c|c|c|c|}
\hline \multirow[t]{2}{*}{$\begin{array}{l}\text { Situation des points } \\
\text { de calculs ou de } \\
\text { mesures }\end{array}$} & \multicolumn{3}{|c|}{$\begin{array}{l}\text { Flux obtenus à partir } \\
\text { des données CARTOQE } \\
\text { sans auto-épuration } \\
(K=1,0)\end{array}$} & \multicolumn{3}{|c|}{$\begin{array}{c}\text { Flux obtenus à partir } \\
\text { des données CARTOQE } \\
\text { avec auto-épuration } \\
(K=0,90) \text { pour les affluents } \\
\text { et }(K=0,95) \text { pour le cours } \\
\text { principal }\end{array}$} & \multicolumn{3}{|c|}{$\begin{array}{c}\text { Flux mesurés au cours } \\
\text { de l'étude } \\
\text { IRAP - } 1985\end{array}$} \\
\hline & $\begin{array}{l}\text { Débitde } \\
\text { référence } \\
\text { d'étiage } \\
\mathrm{m}^{3} / \mathrm{s}\end{array}$ & $\begin{array}{c}\text { Flux } \\
\mathrm{kg} / \mathrm{j} \\
\mathrm{DB} 05\end{array}$ & $\begin{array}{l}\text { Classe de } \\
\text { qualité } \\
\text { obtenue }\end{array}$ & $\begin{array}{l}\text { Débitde } \\
\text { référence } \\
\text { d'étiage } \\
\mathrm{m}^{3} / \mathrm{s}\end{array}$ & $\begin{array}{c}\text { Flux } \\
\mathrm{kg} / \mathrm{j} \\
\mathrm{DBO} 5\end{array}$ & $\begin{array}{c}\text { Classe de } \\
\text { qualité } \\
\text { obtenue }\end{array}$ & $\begin{array}{l}\text { Débit } \\
\text { mesuré } \\
\mathrm{m}^{3} / \mathrm{s}\end{array}$ & $\begin{array}{c}\text { Flux } \\
\mathrm{kg} / \mathrm{j} \\
\mathrm{DBO} 5\end{array}$ & $\begin{array}{c}\text { Classe de } \\
\text { qualite } \\
\text { obtenue }\end{array}$ \\
\hline $\begin{array}{l}92000 \text { Amont Thones } \\
\text { Aval confluence } \\
\mathrm{R}^{\text {au }} \text { de Chantemerle }\end{array}$ & 0.195 & 25 & $1 \mathrm{~A}$ & 0,195 & 25 & $1 \mathrm{~A}$ & - & - & - \\
\hline $\begin{array}{l}91000 \text { Aval Thones } \\
\text { Pont des Morettes }\end{array}$ & 0,500 & 1005 & 3 & 0,500 & 670 & 3 & - & - & - \\
\hline $\begin{array}{l}90000 \\
\text { Pont de Dingy }\end{array}$ & 1,095 & 1110 & 3 & 1,095 & 595 & 2 & - & - & - \\
\hline $\begin{array}{l}89000 \\
\text { Pont d'Onnex }\end{array}$ & 1,66 & 1200 & 2 & 1,66 & 535 & $1 \mathrm{~B}$ & - & - & - \\
\hline $\begin{array}{l}88000 \\
\text { Pont de Brogny }\end{array}$ & 1,84 & 1580 & 2 & 1,84 & 595 & $1 \mathrm{~B}$ & 3,0 & 550 & $1 \mathrm{~A}$ \\
\hline $\begin{array}{l}85000 \text { bis } \\
\text { Usine de Chavaroche }\end{array}$ & 3,42 & 5900 & 3 & 3,42 & 3050 & 2 & 5,3 & 2340 & 2 \\
\hline $\begin{array}{l}84000 \\
\text { Pont d'Hauteville }\end{array}$ & 3.5 & 6010 & 3 & 3.5 & 2450 & 2 & 5.5 & 1200 & $1 \mathrm{~A}$ \\
\hline
\end{tabular}




\section{Conclusion}

L'outil qu'est (ou qu'a été, puisque tous les départements de notre région sont actuellement dotés d'une carte d'objectifs de qualité) CARTOQE attirera sans doute les critiques, voire les sourires des perfectionnistes, dont l'objectif est la meilleure représentation possible de ce qui se passe dans la réalité la plus complexe.

L'objectif poursuivi par CARTOQE était au contraire de simplifier (outrageusement ?) cette réalité pour permet- tre néanmoins, malgré le peu de données disponibles, de tracer un cadre général et cohérent pour la gestion de la qualité des eaux des rivières.

Les résultats tirés de cette expérience sont globalement positifs, puisque 5 cartes départementales ont utilisé cet outil. Son utilisation doit cependant être strictement circonscrite à son objet, c'est-à-dire, la simulation de l'impact sur le milieu de différents niveaux de traitement de la pollution dans les conditions des débits de référence d'étiage.

\section{Annexe 1}

CARTOQE

Exemple d'étude des classes de qualité : Le Fier (Haute-Savoie) Evolution du paramètre DBO5

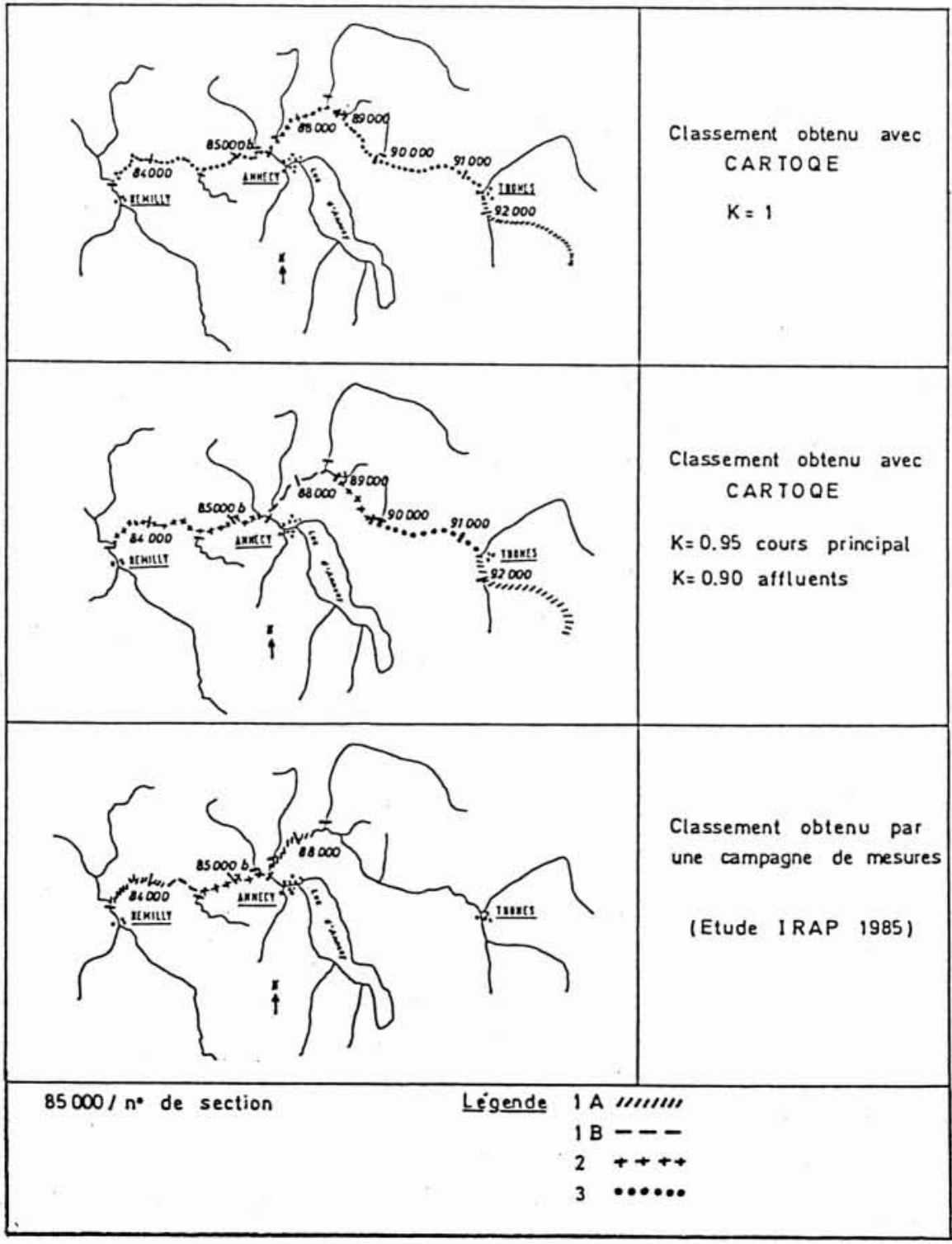




\section{Annexe 2}

\section{CARTOQE}

Exemple de listing de sortie

\begin{tabular}{l} 
!RESURTATS DES SINUSATIONS S.R.A.E. RHONE-ALPES! \\
\hline
\end{tabular}

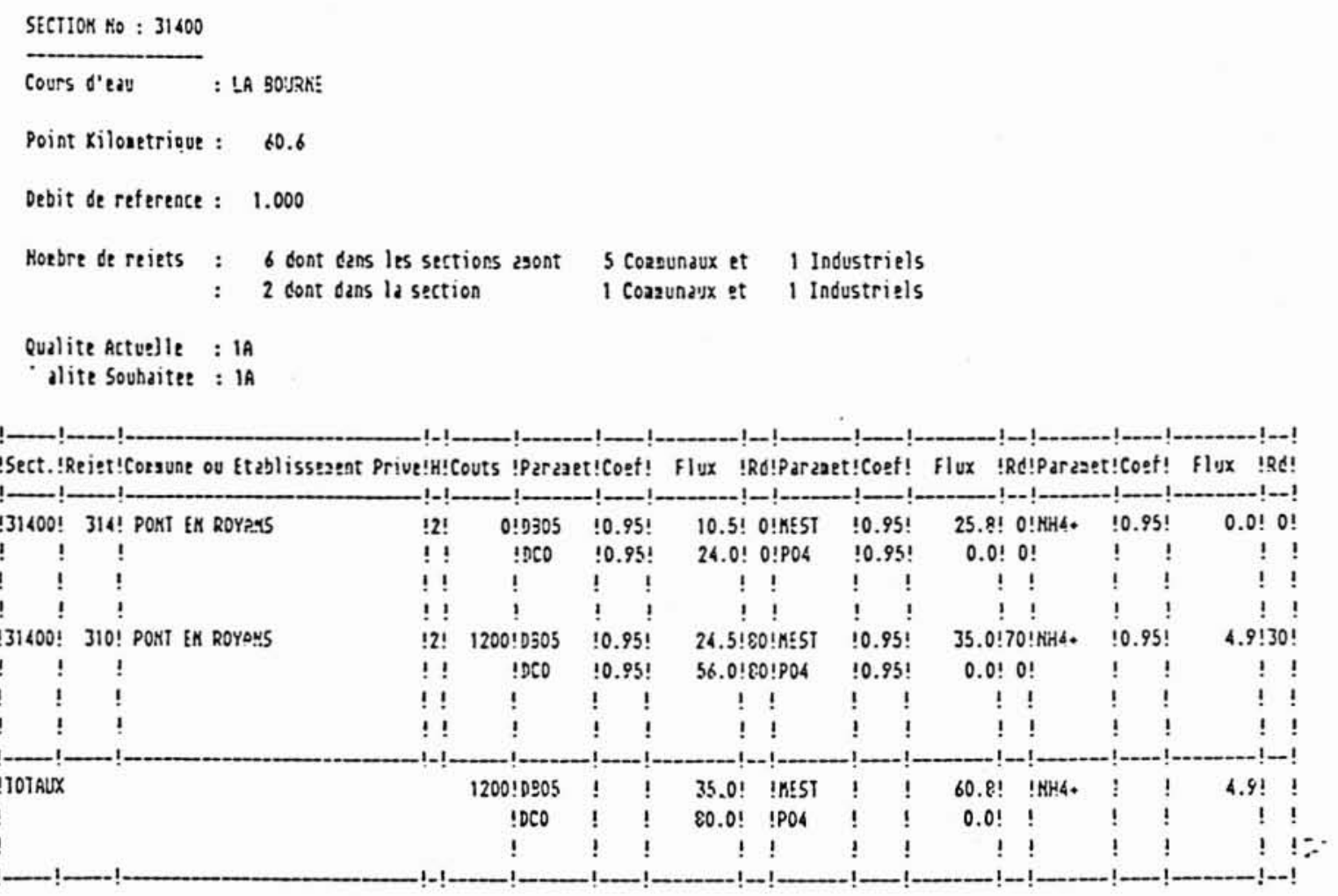

. AATIOK RESULTRKTE AL KIUEAU DE LA SECTIOK 31400

FLIX TOTAL

FLUX DE BRUII DE FOKD

FLUX DE LA SECTIOK AROKT Apport de la Section

\section{CLASSE DE OUALITE OBTENUE}

FLUX DISPOKIBLE PAR RAPPORT AU PLAFOKD DE LA CLASSE

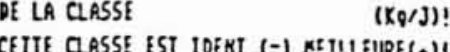
PLUS KAUVAISE(-) OUE LA SECTIOY ESOKT

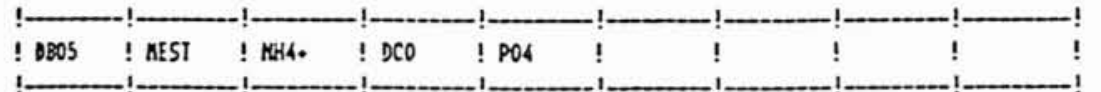

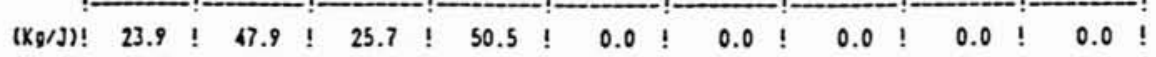

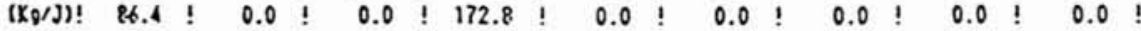

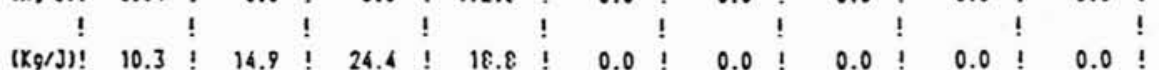

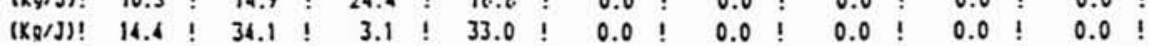

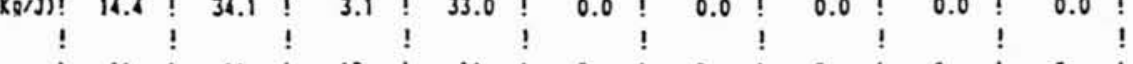

IA ! $1 A$ ! IS ! IA !

148.9 ! $2544.1 ! 17.5$ ! $1504.7 !$

$=\begin{gathered}! \\ ! \\ !\end{gathered}=\frac{1}{!}=1=$

